\title{
Pertussis outbreak in northwest Ireland, January - June 2010
}

A S Barret (annesophie.barret@hse.ie)1,2, A Ryan³, A Breslin33, L Cullen³, A Murray³, J Grogan4, S Bourke5, S Cotter

1. Health Protection Surveillance Centre (HPSC), Dublin, Ireland

2. European Programme for Intervention Epidemiology Training (EPIET), European Centre for Disease Prevention and Control (ECDC), Stockholm, Sweden

3. Department of Public Health, Health Services Executive North West, Sligo, Ireland

4. Our Lady's Sick Children's Hospital (OLSCH), Crumlin, Dublin, Ireland

5. Aghadark General Practice, Ballinamore, Ireland

Citation style for this article:

Barret AS, Ryan A, Breslin A, Cullen L, Murray A, Grogan J, Bourke S, Cotter S. Pertussis outbreak in northwest Ireland, January - June 2010. Euro Surveill.

2010;15(35):pii=19654. Available online: http://www.eurosurveillance.org/ViewArticle. aspx?Articleld=19654

We report a community pertussis outbreak that occurred in a small town located in the northwest of Ireland. Epidemiological investigations suggest that waning immunity and the absence of a booster dose during the second year of life could have contributed to the outbreak. The report also highlights the need to reinforce the surveillance of pertussis in Ireland and especially to improve the clinical and laboratory diagnosis of cases.

\section{Introduction}

Pertussis is a notifiable disease in Ireland. The pertussis case definition used in Ireland includes compatible clinical symptoms alone (possible), clinical symptoms in combination with epidemiological link to laboratory confirmed case (probable) or laboratory confirmation (confirmed). Between 2005 and 2009, on average 81 cases of pertussis were notified annually nationwide. This corresponds to an average incidence rate of 1.9/100,000 population. The majority of cases were children aged less than six months ( $51 \%$ of all cases notified from 2005 to 2009). The Irish vaccination schedule comprises an acellular pertussis-containing vaccine at two, four and six months and a booster dose given when children are between four and five years old.

On 21 April 2010, a general practitioner (GP) in a small town in the north western region of the country notified the Department of Public Health (DPH) - Health Services Executive (HSE) North West of an increase in the number of patients presenting with pertussis-like illness. The age range was broad and the GP reported that the patients were mostly up-to-date with their vaccinations. On 19 May, the first case was confirmed by serology. The DPH launched an epidemiological investigation in order to manage the outbreak, describe the magnitude of the outbreak and develop a hypothesis regarding the cause of the outbreak.

\section{Epidemiological and microbiological investigations}

A possible case was defined as a person living or working in the small town or in the immediate area (consisting of 11 electoral divisions with a total population of 3,624 ) who was diagnosed with pertussis by a GP or who met the European Union/World Health Organization (EU/WHO) clinical case definition for pertussis i.e. cough lasting at least two weeks with one of the following: paroxysms of coughing, inspiratory whoop or post-tussive vomiting between January and June 2010. A probable case was defined as a person who met the possible case definition and had close contact with a confirmed case. A confirmed case was a person who met the possible case definition and had laboratory evidence of Bordetella pertussis infection (isolation of $B$. pertussis from clinical specimen, detection of nucleic acid or demonstration of a specific antibody response in absence of recent vaccination).

We undertook active case finding by contacting all GPs working in the surrounding area, as well as emergency and paediatric departments of the two nearest regional hospitals. For all cases notified by a GP, the presence of other cases in the close circle of family and friends was explored.

The contacted GPs and hospitals were asked to collect a swab specimen or a serum sample from any new possible case of pertussis that presented. GPs do not normally have the naso-pharyngeal swabs that are used for pertussis in their surgery. These swabs were supplied to them along with recommendations for collection and transportation. For serology testing, we used a single high titre of anti-pertussis toxin immunoglobulin G (IgG). The cut-off was set at $100 \mathrm{IU} / \mathrm{ml}$. The laboratory where polymerase chain reaction (PCR) was carried out performed real time PCR testing (IS481-PCR) and also tested for $B$. pertussis toxin promoter and for $B$ parapertussis (insertion sequence). 
We interviewed all cases by telephone using a standardised questionnaire. We collected socio-demographic information, work/educational setting, clinical symptoms, complications secondary to pertussis, outcome and laboratory testing. We gathered vaccination history either from the Local Health Office records (for children under the age of 15 who were vaccinated locally), directly from the cases, or from the notifying GP.

Age-specific attack rates were calculated using 2006 census data [1].

\section{Control measures}

Letters were sent to local GPs, emergency and paediatric departments in the two regional hospitals and local ante-natal clinics in order to inform them about the outbreak and give advice about clinical features, treatment, chemoprophylaxis for vulnerable contacts and vaccination as recommended in the Immunisation Guidelines for Ireland [2].

Letters were also sent to local schools and crèches containing information about the outbreak and recommending that all children should be up-to-date with their vaccinations. Following the notification of a confirmed case in a maternity ward, letters were sent to all those who had been inpatients on the maternity ward at the same time.

\section{Results}

A total of 69 cases were identified from notification data and subsequent active case finding. Two cases were excluded because they were not reported by a GP and did not meet the clinical case definition. Finally 67 cases were included in the analysis. Four of them were probable cases, three were confirmed and the remainder were possible.

Of these 67 cases, 62 were notified by four different GPs (from two GP practices) and five further cases were identified in the close circle of notified cases.

Pertussis testing was carried out in four different laboratories. Seventeen patients were tested in total and three were confirmed (one by culture and two by

\section{FIGURE 1}

Number of cases by week of symptom onset, northwest Ireland, January-June $2010(\mathrm{n}=58)$

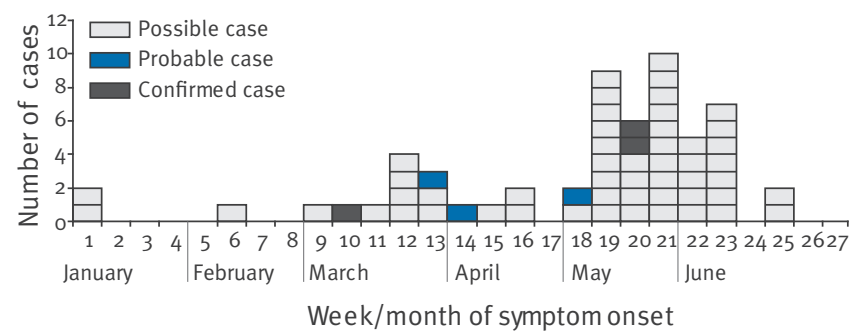

serology). Of seven patients tested by serology, two had a positive result. Of ten patients tested by culture, one had a positive result. Four patients who tested negative by culture were further tested by PCR and all of them had a negative result. Of three negative serology tests for which the information was available, two were taken seven days after symptom onset and one was taken eight days after symptom onset. Of nine negative on culture, one had been taken more than one week after symptom onset and five had been taken more than 14 days after symptom onset.

Of 58 cases for whom the information was available, onset of cough was reported from January to June 2010 with a peak in week 21 (Figure 1).

Four cases were hospitalised in the local hospital: three children aged between four days and one month and one 60 year-old adult. This last case was hospitalised for pneumonia secondary to pertussis.

The male/female ratio was 0.6 . Sixteen cases were aged between one and four years old which corresponds to an attack rate of 77 per 1,000 population. Another 16 cases were aged 10-14 years (attack rate:76/1,000 population). Twenty (30\%) cases were older than 19 years (attack rate:7.6/1,000 population). Considering the number of cases by single year of age, the highest attack rates were in children aged under 12 months (130/1,000 population) and three years (149/1,000 population).

Figure 2 shows the number of cases by age group and the vaccine doses they received. Of five children aged less than six months, one child had received the recommended three priming doses.

Of 17 children aged between six months and four years, 15 had received the recommended priming doses. Of 22 children aged between five and 18 years old for whom the information was available, 20 had received the recommended four doses of vaccine. Vaccination status was unknown for all adults.

Of 51 cases who were interviewed more than 14 days after the cough onset, 50 reported a cough lasting more

\section{FIGURE 2}

Distribution of cases by age group among children aged $\leq 18$ years old, northwest Ireland, January-June 2010 $(\mathrm{n}=47)$

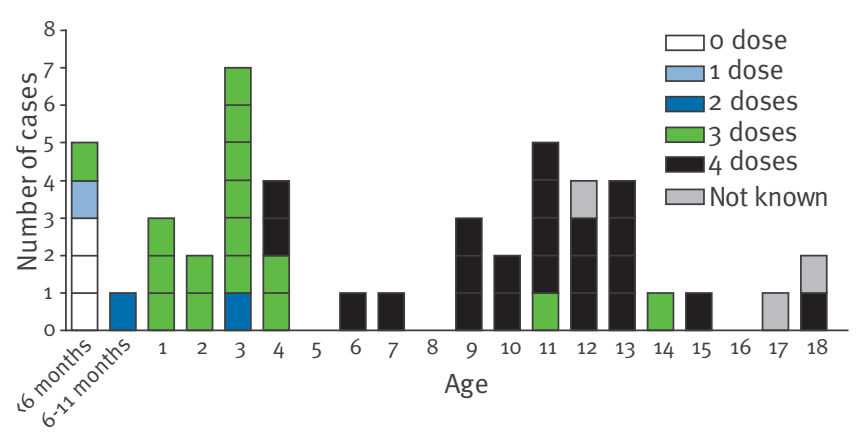


than 14 days (median: 29 days, range: 14-99 days). of 64 cases for whom the information was available, the most frequent reported symptom was paroxysmal cough (52 of 64). Reported symptoms varied according to age. Inspiratory whoop, post-tussive vomiting and apnoea were reported more frequently in children less than one year old than in the other age groups (Figure 3).

\section{Discussion}

In the light of these preliminary results, this outbreak seems to have occurred in a well-vaccinated community and to have spread mostly among children under six months of age, between one and four years old and among the 10-14 year-olds.

There may be more than one factor that contributed to the outbreak. As suggested by the analysis of vaccination data, waning immunity could explain the high number of adolescent cases. We could not verify this hypothesis in adult cases but waning immunity might also have contributed to infection in adults, among other factors.

In the 1990 and 2000 s, findings from various pertussis surveillance systems demonstrated a change in the age profile of pertussis cases in countries with high vaccine coverage rates in young children [3-5]. A shift in the age group has been observed, with increasing pertussis incidence among adolescents and adults. The increase was attributed to improvements in the diagnosis and reporting of adolescent and adult cases, combined with waning immunity. The introduction of an immunisation programme reduces the amount of $B$. pertussis that is circulating in the population. This will result in less natural boosting of immunity amongst those whose immunity is waning. This waning of immunity has led to a recommendation in many countries for a vaccine booster dose for adolescents and adults. The duration of immunity is estimated to range from seven to 20 years after infection with $B$. pertussis and 4-12 years after vaccination with whole or acellular pertussis vaccine [6-8], thus making adolescents and adults more susceptible to pertussis disease. Waning immunity and the subsequent increase in disease is associated with considerable morbidity and economical costs and increases the probability of transmission of pertussis infection to vulnerable children. Various studies have shown that adolescent siblings and parents are often the major source of transmission of pertussis to infants $[5,8,9]$.

During the outbreak, five infants aged less than six months were diagnosed with pertussis. For three of them, a symptomatic contact was reported with onset of illness prior to their own (cousin and mother). It is likely that these contacts had transmitted pertussis to these infants.

Following the awareness of waning immunity, a booster dose at adolescence was introduced in the vaccination schedule in the United States (US), Canada and many European countries [10]. In Ireland, a booster dose is recommended since 2008 but has not been routinely provided through the national immunisation programme (usually administered in schools).

On the other hand, we observed a high incidence rate among children aged between one and four years old despite good vaccination coverage Most recent data available on immunisation coverage ( $Q_{1}$ 2010) indicates that the vaccination coverage rate at both 12 and 24

\section{FIGURE 3}

Distribution of reported symptoms by age group, northwest Ireland, January-June 2010 (n=64)

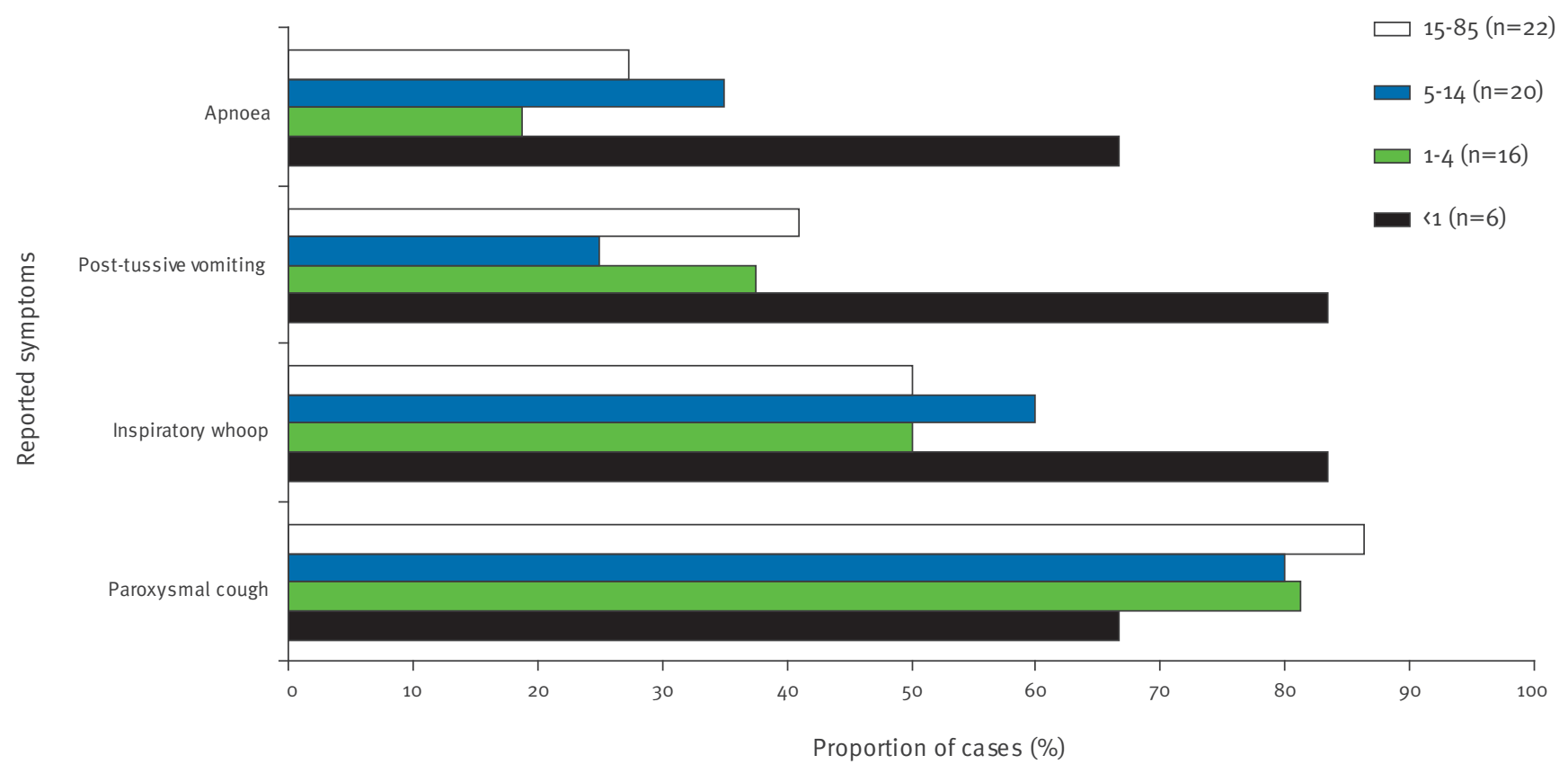


months (93\% and $97 \%$ ) in the HSE North West region exceeds national average ( $89 \%$ and $94 \%$ respectively). Effectiveness of acellular vaccines in infants has been estimated to be high (71\%-93\%) [11]. Since 1996 acellular pertussis vaccines have been used in the childhood vaccination programme. The vaccine used since September 2008 (INFANRIX- HEXA).is a three-component pertussis-containing vaccine. Two vaccines are currently used in Ireland for the booster dose, either a two-component pertussis containing vaccine (TETRAVAC) or three-component pertussis vaccine (INFANRIX-IPV). Both vaccines are licensed for booster usage. In this investigation we did not seek information on the vaccine brand used. However, as this outbreak affected many age cohorts we do not have reason to suspect low vaccine effectiveness linked to a specific batch of vaccine as different vaccines and batches would have been used for the population affected. A more likely explanation may be waning immunity due to the absence of a booster dose in the second year of age. But as Ireland has never had a booster at this age it is not evident why this large outbreak should be occurring now, in this highly vaccinated population.

The duration of protection after the priming doses at two, four and six months is not clear [9]. Current vaccination schedules in the US, Canada and all European countries except the United Kingdom and Ireland include a booster dose between 10 and 24 months. At a recent meeting of the Strategic Advisory Group of Experts on immunisation (SAGE) of the WHO in April 2010, experts stated a preference for giving a pertussis booster dose in the second year of life [12].

The possibility that other pathogens, respiratory syncytic virus (RSV) and adenovirus, may have contributed to some of the cases reported cannot be ruled out. No samples from patients involved in this outbreak were sent for virological investigation. But outbreaks associated with these pathogens were not reported from other parts of the country and during this period the prevalence of RSV from respiratory samples taken at non-sentinel GP sites in Ireland was low, with just $5.4 \%$ of all samples positive for RSV, in comparison to $14.1 \%$ for the same time period in the previous influenza season. The peak period of detection for RSV in the 200910 season was December-January (unpublished data HPSC). The extent and development of this outbreak, together with the clinical presentation and also laboratory confirmation supports our hypothesis that most cases were likely to be pertussis.

An antigenic divergence between the circulating and the vaccine strains, as observed in the Netherlands [13], can not be excluded yet as a contributing factor in the outbreak. Further microbiological investigations are ongoing to explore this hypothesis.

In this outbreak, we found that symptoms varied according to age. In particular, the typical symptoms for pertussis (whoop and post-tussive vomit) are less common among older patients. Pertussis is thought to be underreported as a result of this atypical presentation in adolescents and adults [3]. As previously suggested [3], this highlights the need for a case definition for older individuals in order to improve the reporting. Findings from this outbreak, along with the outbreaks that occurred in California and Australia highlighting the fact that pertussis outbreaks still occur, despite vaccination programmes, and the need to report so that control measures can be taken $[14,15]$, should be communicated to GPs in order to increase their vigilance and their awareness of the symptomatology of pertussis in adolescents and adults.

The large proportion of cases reporting apnoea in this investigation is surprising. The highest proportion was in the youngest age group ( $\$ 1$ year of age), but was also commonly reported in the older age groups. Apnoea associated with pertussis is infrequently reported in older age groups. The question regarding apnoea was asked as interruption of external breathing. Whether patients or parents of children responding to this question understood what was being asked needs to be considered as a reason for this finding as this may reflect a misinterpretation of the question.

One limitation in our findings is the low number of confirmed cases. Ninety percent of those tested by PCR or culture had a negative result. Although there might be some true negative patients, it is likely that some of them were false negatives. Indeed, the sensitivity of laboratory diagnostic methods for pertussis can be affected by various factors such as the sampling technique, timing of sampling since the symptom onset, delay in transporting the specimen, treatment prior to sampling, age and vaccination status $[16,17]$. Culture and PCR have a low sensitivity if the specimen is taken late in the illness. In the outbreak, the late collection of nasopharyngeal swabs may have contributed to the low positivity rate. Problems linked to sampling technique may also explain some negative results.

For serology testing, a single high titre of anti-pertussis toxin IgG has been showed to be useful in late pertussis diagnosis, with a sensitivity of $76 \%$ and a specificity of $99 \%$ using a cut-off of $100 \mathrm{IU} / \mathrm{ml}$ [18]. In the outbreak, some of the negative blood samples were taken within eight days from symptom onset, probably too early to have a detectable level of IgG. Clinicians need guidelines on appropriate samples to be referred for confirmation of pertussis. They should be encouraged to take a sample for culture and PCR at the early stage of infection; whereas serology testing to detect IgG antibodies to pertussis toxin should be recommended when patients present themselves more than two weeks from symptom onset.

The hypotheses that we developed in the descriptive investigation still need to be verified through rigorous analytical study. A retrospective cohort study is currently ongoing among school children in all local 
primary schools. It will allow us to further explore the waning immunity in adolescents, to estimate the vaccine effectiveness and to address other contributing factors.

\section{Conclusion}

This pertussis outbreak occurred in a well-vaccinated community; this is a rare phenomenon that has not been reported in Ireland for many years. We hope that this descriptive study will inform both national vaccination policy and the management of possible future outbreaks. This outbreak was managed by raising awareness in order to promote early diagnosis, treatment and vaccination. Consideration should be given to what role vaccination could have had in preventing this outbreak, whether by giving the recommended adolescent booster or by giving a booster dose earlier than four or five years of age as in other countries. Further epidemiological and microbiological investigations of this outbreak are ongoing regarding the circulating strain of $B$. pertussis, vaccine efficacy and the timing of boosters in relation to infection.

\section{References}

1. Central Statistics Office Ireland [Internet].Cork, Ireland. [updated 2009 Sep 4]. Available from: http://www.cso.ie/ statistics/Population.htm

2. National Immunisation Advisory Committee - Royal College of Physicians of Ireland. Immunisation Guidelines for Ireland Chapter 11. 2008 Edition.

3. Tan T, Trindade E, Skowronski D. Epidemiology of pertussis. Pediatr Infect Dis J. 2005;24(5 Suppl):S10-S18.

4. Guris D, Strebel PM, Bardenheier B, Brennan M, Tachdjian $\mathrm{R}$, Finch $\mathrm{E}$, et al. Changing epidemiology of pertussis in the United States: increasing reported incidence among adolescents and adults, 1990-1996. Clin Infect Dis. 1999;28(6):1230-7.

5. Wirsing von König CH, Riffelman M. Pertussis: An old disease in new clothes. Euro Surveill. 2007;12(9): pii=727. Available from: http://www.eurosurveillance.org/ViewArticle. aspx?Articleld $=727$.

6. Wendelboe AM, Van Rie A, Salmaso S, Englund JA. Duration of immunity against pertussis after natural infection or vaccination. Pediatr Infect Dis J. 2005;24(5 Suppl):S58-S61.

7. Sin MA, Zenke R, Ronckendorf R, Littmann M, Jorgensen $P$, Hellenbrand W. Pertussis outbreak in primary and secondary schools in Ludwigslust, Germany demonstrating the role of waning immunity. Pediatr Infect Dis J. 2009;28(3):242-4.

8. Edwards KM. Overview of pertussis: focus on epidemiology, sources of infection, and long term protection after infant vaccination. Pediatr Infect Dis J. 2005;24(6 Suppl):S104-8.

9. Bisgard KM, Pascual FB, Ehresmann KR, Miller CA, Cianfrini $C$, Jennings CE, et al. Infant pertussis: who was the source? Pediatr Infect Dis J. 2004;23(11):985-9.

10. European Centre for Disease Prevention and Control (ECDC) Guidance. Scientific Panel on Childhood Immunisation Schedule: Diphteria-tetanus-pertussis (DTP) vaccination. 2009 Available from: http://www.ecdc.europa.eu/en/publications/ Publications/o911_GUI_Scientific_Panel_on_Childhood_ Immunisation_DTP.pdf

11. World Health Organization. The Immunological basis for Immunization Series. Module 4: Pertussis. Update 2009. Immunization, Vaccines and Biologicals, WHO; Geneva, 2010. Available from: http://whqlibdoc.who.int/ publications/2010/9789241599337_eng.pdf

12. World Health Organization. Meeting of the Strategic Advisory Group of Experts on immunization, April 2010 conclusions and recommendations. Wkly Epidemiol Rec. 2010;85(22):197-212.

13. Mooi FR, van Loo IH, van GM, He Q, Bart MJ, Heuvelman KJ, et al. Bordetella pertussis strains with increased toxin production associated with pertussis resurgence. Emerg Infect Dis. 2009;15(8):1206-13.
14. Winter K, Harriman K, Schechter R, Yamada E, Talarico J, Chavez G. Pertussis - California, January-June 2010. MMWR Morb Mortal Wkly Rep. 2010;59(26):817.

15. Roper K, Surveillance Branch, Office of Health Protection. Outbreak of pertussis, 1 January to 31 March 2009. Commun Dis Intell. 2009;33(1):36-7.

16. Crowcroft NS and Pebody RG. Recent developments in pertussis. Lancet. 2006;367(9526):1926-36.

17. World Health Organization. Laboratory manual for the diagnosis of whooping cough caused by Bordetella pertussis / Bordetella parapertussis. WHO/IVB/04.14. Immunization, Vaccines and Biologicals. WHO; Geneva, 2004. Available from: http://www.who.int/vaccines-documents/DocsPDFo4/ www788.pdf

18. de Melker HE, Versteegh FG, Conyn-Van Spaendonck MA, Elvers LH, Berbers GA, van Der Zee A, et al. Specificity and sensitivity of high levels of immunoglobulin $\mathrm{G}$ antibodies against pertussis toxin in a single serum sample for diagnosis of infection with Bordetella pertussis. J Clin Microbiol. 2000;38(2):800-6. 